\title{
A Case Report : Pregnancy with Multiple Sclerosis
}

\author{
Shiuly Chowdhury, ${ }^{1}$ Nilufar Islam, ${ }^{2}$ Khadiza Nurun Nahar, ${ }^{3}$ Kaniz Fatema, ${ }^{4}$ Salma Akter Munmun, ${ }^{5}$ Sudip Barua ${ }^{6}$
}

\begin{abstract}
Multiple sclerosis causes disability in young adults and like most autoimmune diseases, affects women more commonly in their child-bearing years. In general, pregnancy does not appear to affect the long-term clinical course of multiple sclerosis and the disease does not affect the course or outcome of pregnancy. In this reportedcase, a pregnant woman with multiple sclerosis showed relapse of the disease in first half of her pregnancy and disease was well controlled in the second half of her pregnancy and postpartum period after taking appropriate measure. Women who have multiple sclerosis and wish to have a family can usually do so successfully with the assistance of their neurologist and obstetrician.
\end{abstract}

Key words: Multiple Sclerosis(MS), Pregnancy, Delivery, Antenatal, Postpartum, corticosteroids.

\section{INTRODUCTION:}

Multiple sclerosis (MS) is the most common inflammatory disorder of the central nervous system during young adulthood. Both genetic and environmental factors are considered to be involved in the pathogenesis of MS. ${ }^{1}$ Unfortunately many obstetricians are not usually experienced with MS. Conversely many neurologists have limited knowledge of obstetrics and pregnancy in MS. ${ }^{2}$

\section{CASE REPORT:}

A 25-year old nulliparous woman presented with a history of 38 weeks pregnancy with premature rupture of membrane with multiple sclerosis (MS). She got herself admitted in the Department of Obstetrics \& Gynaecology in Bangabandhu Sheikh Mujib Medical University (BSMMU). On per abdominal examination, her uterus corresponded to 32 weeks of pregnancy with cephalic presentation of foetus. Clinically it was a case of 38 weeks pregnancy with IUGR (intrauterine growth retardation), oligohydramnios \& PROM (premature rupture of membrane) with MS. Multiple sclerosis was diagnosed, before pregnancy three years back. She had unilateral (hemicranial) headache and had difficulty in movement of eyeball on the right side and fatigue. In the Neurology Department she was diagnosed as a case of multiple sclerosis. The diagnosis was confirmed with medical history, presenting symptoms, physical examination and investigations with lumber puncture and MRI. She was treated with prednisolone, a steroid and anti-inflammatory drug. But she discontinued taking medicine when she got relieved of her symptoms.

She attended at OPD early in her pregnancy. In very early weeks, her pregnancy was uneventful. She was on regular antenatal checkup at OPD of Obstetrics \& Gynaecology Department. Neurologists' opinion was taken time and again. Initially she

\section{Authors' information:}

'Dr. Shiuly Chowdhury, Associate Professor, Department of Obstetrics \& Gynaecology, C-Block (7th floor), BSMMU, Shahbagh, Dhaka-1000 2 Dr. Nilufar Islam, Medical Officer, Department of Obstetrics \& Gynaecology, BSMMU, Shahbagh, Dhaka-1000.

${ }_{3}^{3}$ Dr. Khadiza Nurun Nahar, Associate Professor, Department of Obstetrics \& Gynaecology, C-Block (7th floor), BSMMU, Shahbagh, Dhaka-1000 ${ }^{4}$ Dr. Kaniz Fatema, Associate Professor, Department of Obstetrics \& Gynaecology, C-Block (7th floor), BSMMU, Shahbagh, Dhaka-1000

${ }^{5}$ Dr. Salma Akter Munmun, Medical Officer, Department of Obstetrics \& Gynaecology, BSMMU, Shahbagh, Dhaka-1000

${ }^{6}$ Dr. Sudip Barua, Resident of Neurosurgery, NINS, Agargaon, Dhaka

Correspondence: Dr. Shiuly Chowdhury, Cell Phone: +8801755527698, Email:shiuly07@yahoo.com 
was not put on any medication and was asked to review with them after delivery. Two months later, at around $14^{\text {th }}$ weeks of pregnancy, she experienced headache which was hemicranial and radiating to the right side of the face, teeth and had difficulty in movement of eyeball on the right side and weakness of extremities. Relapse of symptoms of multiple sclerosis compelled her to be admitted in Neurology Department of this university hospital, where she was treated with steroid and other medicines. With improvement of symptoms she was released from the hospital with treatment plan of pulse methylprednisolone at scheduled intervals for rest of her pregnancy period. In the initial months, total 5 doses of injectable methylprednisolone were given and then three doses every month till delivery. The postpartum treatment plan was also there and it included capsule Imuran daily after delivery. Around 24 weeks of pregnancy, she developed gestational diabetes mellitus which was managed with diet and light exercise. In the last trimester, IUGR was diagnosed and the patient was kept under closed supervision for the rest of her pregnancy. At around 38 weeks of pregnancy she presented with premature rupture of membrane with meconium stained liquor. Considering the safety of both mother and child, delivery by caesarean section was planned. The baby developed respiratory distress syndrome after delivery, because of meconium aspiration. The baby needed NICU support for 5 days. With symptomatic improvement, both mother and baby were discharged from the hospital. She was advised to visit Obstetrics and Gynae OPD for postnatal follow up as well as Neurology OPD for follow up of multiple sclerosis. With the continuation of medicine, injection solupred (methylprednisolone) every month and tablet Imuran (Azathioprine, a disease modifying drug), 50 mg three times daily and scheduled follow up in respective OPD, her present health condition is good without relapse.

\section{DISCUSSION:}

Multiple sclerosis is a T-cell mediating autoimmune demyelinating disorder of the central nervous system (CNS) ${ }^{3}$ that preferentially affects women of child-bearing age; approximately $10 \%$ of women first present with symptoms with pregnancy. ${ }^{4}$ Therefore, pregnancy and delivery become an important issue for those with MS. ${ }^{5}$ Previously, MS specialists often advised against child-bearing because of the possible impact of the disease on pregnancy and delivery. ${ }^{6}$ Recently, young adults with MS are being encouraged to raise family. ${ }^{7}$

Fortunately, pregnancy does not appear to speed up the course of the disease or worsen the effects of MS. However, it is thought that women who have unrecognized MS are more likely to begin having symptoms during pregnancy. Some studies have found that MS symptoms decrease in pregnancy and increase during the postpartum (after delivery) period. ${ }^{8}$ It is thought that relapse rate is reduced during the late pregnancy because of the effect of pregnancy hormones which suppress the immune system to ensure that the body will carry a growing baby without rejecting it. Relapse rate after birth may increase because of hormonal disturbances, but there is no consensus about this. ${ }^{9} \mathrm{~A}$ short course of high-dose corticosteroids to hasten recovery from relapses appears relatively safe during pregnancy, but generally should be limited to disabling relapses. Often there is a possible increased risk of fetal cleft palate associated with corticosteroid treatment in the first trimester. ${ }^{10}$ Relapses during pregnancy can be treated with corticosteroids but caution is advised prior to gestational week 12 because of the risk of cleft palate. In the case of severe relapse in the first trimester, the preferred treatment is prednisolone as it is inactivated in the placenta. ${ }^{11}$

Women in labour may not have pelvic sensation and may not feel pain with contraction. Delivery of the fetus may be difficult. While labour itself is not affected, the muscles and nerve needed for pushing can be affected. This may make caesarean section, forceps and vacuum assisted deliveries more likely. ${ }^{8}$ More frequent prenatal visits may be needed. In this case baby suffered from intrauterine growth retardation (IUGR) and the mother had gestational diabetes mellitus 
during antenatal period. She also had premature rupture of membrane. The safety of both mother and baby at delivery is paramount in such patients. In the present case, delivery by caesarean section was done for obstetric indication. However, medications like steroid may be used in pregnancy if needed. Breast feeding was not associated with relapse. In the present case, there was relapse of the disease in the first trimester of pregnancy and general health status was stable during the third trimester as well as in the postpartum period with the continuation of steroid and immunosuppressive therapy as scheduled.

\section{CONCLUSION:}

Pregnant women with MS need close monitoring of the disease for maternal and foetal wellbeing. Clinical course as well as relapse rate in early pregnancy and postpartum period can be reduced if the patient could be kept under close monitoring.

\section{REFERENCES:}

1. Compstona, Coles A. Multiple sclerosis. Lancet 2008; 372(9648):1502-1517

2. Borisow N, Paul F, Ohlraun S, Pach D, Fischer F, Dorr J. Pregnancy in Multiple Sclerosis: a questionnaire study. PloS One 2014;9(6):e99106

3. Houchens MK. Pregnancy and multiple sclerosis. Semin Neurol 2007; 27:434-441.

4. Bennet KA. Pregnancy \& multiple sclerosis. Clin Obstet Gynecol 2005;48:38-47.

5. R Bove T. Chitnis The role of gender and sex hormones in determining the onset and outcome of multiple sclerosis. Mult Scler 2014;20:520-526.

6. Sadovnick $A D$, EisenK, Hashimoto $S A$, Farquhar $R$, Yee IM, Hooge J, etal.Pregnancy and multiple sclerosis. A prospective study. Arch Neurol 1994;51:1120-124.

7. Bove R, Alwan S, Friedman JM, Hellwig K, Houtchens M, Koren $\mathrm{G}$, et al. Management of multiple sclerosis during pregnancy and the reproductive years: a systematic review. Obstet Gynecol 2014;124(6):115768. doi: 10.1097/AOG.0000000000000541.

8. Multiple Sclerosis and Pregnancy-Stanford Children's Health https://www.stanfordchildrens.org/en/topic/default? id =multiple-sclerosis... pregnancy.
9. www. Mstrust.org.uk/a-z/pregnancy

10. Park-Wyllie L, Mazzotta P, Pastuszak A, et al. Birth defects after maternalexposure to corticosteroids: Prospective cohort study and meta-analysis of epidemiological studies. Teratology 2000; 62: 385392.

11. Kerstin Hellwig: Pregnancy in Multiple Sclerosis. Eur Neurol 2014;72(suppl 1):39-42. DOI: 10.1159/ 000367640. 